January 30, 2009

\title{
Mode locking in a spatially extended neuron model: active soma and compartmental tree
}

\author{
C-M Svensson and S Coombes \\ School of Mathematical Sciences, University of Nottingham, \\ University Park, Nottingham, NG7 2RD, UK.
}

January 30, 2009

\begin{abstract}
Understanding the mode-locked response of excitable systems to periodic forcing has important applications in neuroscience. For example it is known that spatially extended place cells in the hippocampus are driven by the theta rhythm to generate a code conveying information about spatial location. Thus it is important to explore the role of neuronal dendrites in generating the response to periodic current injection. In this paper we pursue this using a compartmental model, with linear dynamics for each compartment, coupled to an active soma model that generates action potentials. By working with the piece-wise linear McKean model for the soma we show how the response of the whole neuron model (soma and dendrites) can be written in closed form. We exploit this to construct a stroboscopic map describing the response of the spatially extended model to periodic forcing. A linear stability analysis of this map, together with a careful treatment of the non-differentiability of the soma model, allows us to construct the Arnol'd tongue structure for 1: $q$ states (one action potential for $q$ cycles of forcing). Importantly we show how the presence of quasi-active membrane in the dendrites can influence the shape of tongues. Direct numerical simulations confirm our theory and further indicate that resonant dendritic membrane can enlarge the windows in parameter space for chaotic behavior. These simulations also show that the spatially extended neuron model responds differently to global as opposed to point forcing. In the former case spatio-temporal patterns of activity within an Arnol'd tongue are standing waves, whilst in the latter they are traveling waves.
\end{abstract}




\section{Introduction}

Neurodynamical models based on spiking neurons are playing an increasing role in the interpretation of neurophysiological data. Neurons uses a variety of coding mechanisms to represent stimuli. The most prominent of these being spike and rate based [Dayan and Abbott, 2001]. Importantly the precise timing of action potential firing events that can be generated by biological neurons is thought to underlie several different forms of sensory processing [Rieke et al., 1997, Hunter et al., 1998]. The role of precisely timed spikes has also been shown to have importance in higher brain regions such as cortex and neo-cortex

[Azouz and Gray, 2000, Fellous et al., 2001, Mainen and Sejnowski, 1995]. To probe the nature of the neural spike code it is natural to consider experiments whereby a single neuron is forced with a periodic signal. In the case of the squid giant axon

[Aihara et al., 1986, Kaplan et al., 1996], spinal interneurons [Beierholm et al., 2001] and cortical pyramidal cells [Brumberg and Gutkin, 2007] this has been shown to lead to socalled mode-locked responses. Briefly these characterize responses describing $p$ periods of oscillation per $q$ periods of the forcing. One area in particular where neural spike timing on a millisecond time scale and mode-locking have been linked is in the study of mechanoreceptor responses to periodic vibrations [Freeman and Johnson, 1982]. In some instances this periodic forcing can be thought of as emulating real sensory input or input from other brain regions. An example of the latter would be hippocampal cells driven by the theta rhythm [Huhn et al., 2005, Lengyel et al., 2003]. A detailed mathematical framework for understanding mode-locking in point oscillator models now exists, (see [Glass and Mackey, 1988, Pikovsky et al., 2001] for an overview), although the extension to spatially extended systems is still in its infancy [Lin et al., 1999]. The techniques for understanding mode-locking in point oscillators have now been applied to several neuron models [Nagumo and Sato, 1972, Yoshino et al., 1999] including variants of the popular integrate-and-fire (IF) model such as the leaky IF model [Keener et al., 1981, Coombes and Bressloff, 1999], the IF-or-burst model [Coombes et al., 2001] and the "ghostburster" model [Laing and Coombes, 2005]. These particular IF models do not attempt to mimic the shape of the action potential (AP) in a real neuron. The study of mode-locking in a conductance based Hodgkin-Huxley model [Aihara et al., 1984], capable of generating a realistic AP shape, is challenging due to the complexity of the underlying single neuron model. Indeed mathematical 
progress is typically only possible with the use of a numerically constructed Poincare map, as in the work of [Lee and Kim, 2006]. The simpler FitzHugh-Nagumo (FHN) model suffers from a similar lack of analytical tractability

[Sato and Doi, 1992, Doi and Sato, 1995]. However, the McKean model [McKean, 1970] provides a useful piece-wise linear (PWL) caricature of the FHN model that has allowed for some analysis, at least in the singular limit [Coombes and Osbaldestin, 2000]. Although PWL models that can mimic the shape of an AP, such as the McKean model, are natural models of the neuronal cell body (soma) they do not provide any representation of the dendritic tree. These branched structures can occupy up to $90 \%$ of the volume of the cell and as such can have a significant affect on the response of the cell [Stuart et al., 2008]. Compartmental modeling, utilizing a system of coupled ordinary differential equations, is a powerful framework for describing the electrical properties of realistic dendritic trees [Rall, 1964]. Here we combine PWL models of excitable media with compartmental modeling to develop a more realistic description of the spatially extended neuron. Importantly we show how to construct mode-locked responses of this model cell in closed form, without the need to work in the singular limit. We use this machinery to unravel the response of a spiking neuron to input that is either at a point on the dendritic tree or applied globally.

In Sec. 2 we describe the McKean model and a simple dendritic tree in the form of a finite number of nearest-neighbor coupled compartments. In the case of a passive compartment (for an RC circuit) there is only one linear ODE for each compartment, whilst for the more general case describing so-called quasi-active membrane [Koch, 1984] (for an LRC circuit) there are at least two. Since the McKean model is PWL and the dendritic model is linear the combined soma-dendrite model is PWL. The general solution to arbitrary spatio-temporal forcing is given in closed form using matrix Green's functions. Next in Sec. 3 we focus on the case of period temporal forcing, which is either global (applied to all compartments) or local (applied to a single compartment). Mode-locked solutions are constructed in terms of the solution to a set of coupled nonlinear algebraic equations. These are obtained by matching solutions to the PWL soma-dendrite model at points where the somatic voltage crosses one of two thresholds, as well as from enforcing periodicity. To probe the spatio-temporal behavior of mode-locked states throughout the dendritic tree we use a Fourier technique to plot the spatial phase of the pattern. These phase-plots have very different topologies for standing and traveling waves, and easily allow one to visualy identify which of the 
two cases is manifested. The stability of mode-locked solutions is determined in Sec. 4 . Borders in parameter space defining bifurcations are defined in terms of both the instabilities of a stroboscopic map and grazing bifurcations of the somatic voltage with one of the two thresholds. Knowledge of these instabilities allows us to calculate the Arnol'd tongue structure for the model. This is compared with direct numerical simulations and shown to be in excellent agreement. Finally in Sec. 5 we discuss extensions of the work in this paper.

\section{The Model}

In previous work it has been shown that there is a planar PWL model that can provide a quantitative fit to the Hodgkin-Huxley model [Chik et al., 2004]. For clarity of exposition we will focus on a special case - the McKean model - that is easily re-parametrised along the lines of Chik et al. if desired. Indeed, the McKean model [McKean, 1970] is itself a PWL caricature of the planar FHN model [Fitzhugh, 1961] of a neuron. The FHN model is written in the form

$$
\begin{aligned}
c \dot{v} & =f(v)-w+J, \\
\dot{w} & =v-\gamma w,
\end{aligned}
$$

where $v$ is the somatic voltage, $w$ is a recovery variable, and $J$ is an injected current. Here $c$ represents the capacitance of the membrane and $\gamma>0$. The FHN model has a cubic voltage nullcline and a linear nullcline for its recovery variable. To obtain the McKean model the cubic nullcline of the FHN model is simplified by the replacement of the cubic function $f$ with

$$
f(v)= \begin{cases}-v, & v<a / 2 \\ v-a, & a / 2<v<(1+a) / 2 \\ 1-v, & v>(1+a) / 2 .\end{cases}
$$

The simplicity of the McKean model means that periodic solutions describing APs can be written in closed form [Tonnelier, 2002, Coombes, 2008]. In Fig. 1 we plot the phase plane of the McKean model and a typical periodic orbit.

It is well known that the electrical properties of a dendritic tree allow for the diffusive spread of current from the point of injection. The passive nature of this communication has led to the development of the cable equation (a second-order linear 
partial differential equation) for the dendrite. An alternative approach is to view the tree as a graph of connected electrical compartments [Rall, 1964]. The resulting system of coupled linear ODEs is often regarded as a finite difference approximation of the cable equation, in which the dendritic system is divided into sufficiently small regions such that spatial variations of the electrical and physical properties within a region are negligible. Interestingly, many neurons exhibit resonances, arising from nonlinear ionic conductances, whereby subthreshold oscillatory behavior is amplified for inputs at preferential frequencies. Such resonant dynamics has been shown for many types of neurons [Hutcheon and Yarom, 2000], for example in rat sensimotor cortex [Hutcheon et al., 1996] and CA1 pyramidal cells where subthreshold oscillations have been connected to associative memory processes [Graham, 2004]. The linearisation of active currents about rest, can often account for the observed resonant dynamics [Koch, 1984, Coombes et al., 2007]. In the terminology of electrical engineering the resulting linear system has a membrane impedance that displays resonant-like behavior due to the additional presence of inductances. This extends the more usual ' $R C^{\prime}$ ' circuit description of passive membrane to the so-called quasi-active or 'LRC' case. For a compartmental chain with quasi-active membrane we follow [Bressloff and Coombes, 1997, Bressloff, 1999] and write

$$
\begin{aligned}
C \frac{\mathrm{d} V_{i}}{\mathrm{~d} t} & =-g V_{i}+\widetilde{g} \sum_{j \in \mathrm{nn}}\left(V_{j}-V_{i}\right)-I_{i}+I_{\mathrm{inj}, i}(t), \\
L \frac{\mathrm{d} I_{i}}{\mathrm{~d} t} & =-r I_{i}+V_{i}, \quad i=1, \ldots, N .
\end{aligned}
$$

These equations represent a set of $N$ identical ' $L R C$ ' circuits coupled by nearest-neighbor $(\mathrm{nn})$ interactions only. In the voltage equation (4) $C$ represents capacitance, $g$ leakage conductance or reciprocal resistance $R=1 / g, \widetilde{g}$ the coupling conductance between neighboring compartments, $I_{\mathrm{inj}, i}$ the injected current to the ith compartment, and $I_{i}$ the current arising from quasi-active membrane. This current obeys the linear equation (5), where $L$ is an inductance and $r$ a resistance. All the electrical parameters $(L, R, C, r)$ of a single compartment, depicted in Fig. 2, can be derived from biophysical models of Hodgkin-Huxley type, as described in [Koch, 1984]. Note that to obtain a purely passive ' $R C^{\prime}$ model we simply take the limit $r \rightarrow \infty$ so that no current can flow through the inductive branch of the compartmental circuit.

To build a model that combines the McKean soma model with the compartmental tree model we simply add a coupling term $\widehat{g}\left(V_{1}-v\right)$ to the right hand side of (1), and a 
term $\widehat{g}\left(v-V_{1}\right)$ to the right hand side of $(4)$ for $i=1$. Here $\widehat{g}$ is the coupling conductance between the soma and the dendrite. The $2 N+2$ ODEs defining the soma-dendrite model are PWL. A schematic of this soma-dendrite model is shown in Fig. 3. Defining two voltage thresholds $v_{\mathrm{th}}^{1}$ and $v_{\mathrm{th}}^{2}$ by $\left(v_{\mathrm{th}}^{1}, v_{\mathrm{th}}^{2}\right)=(a / 2,(1+a) / 2)$, then in any region of phase space away from the borders where $v=v_{\text {th }}^{1,2}$ the dynamics is described simply by $2 N+2$ linear ODEs, which we may write as

$$
\dot{z}=A z+b(t), \quad z=\left[\begin{array}{llllllll}
V_{1} & \ldots & V_{N} & I_{1} & \ldots & I_{N} & v & w
\end{array}\right]^{T} .
$$

The $(2 N+2) \times(2 N+2)$ matrix $A$ has the block form

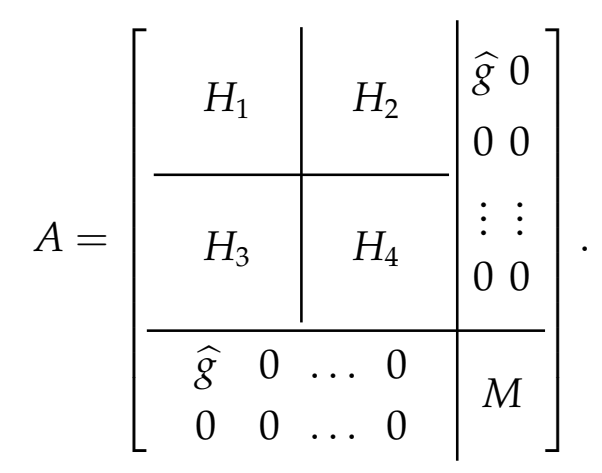

Here the $N \times N$ matrices $H_{i}, i=1, \ldots, 4$, are given explicitly by

$$
H_{1}=\left[\begin{array}{cccccc}
-(g+\widehat{g}+2 \widetilde{g}) / C & \widetilde{g} / C & 0 & 0 & \ldots & 0 \\
\widetilde{g} / C & -(g+2 \widetilde{g}) / C & \widetilde{g} / C & 0 & \ldots & 0 \\
\ldots & \ldots & \ldots & \ddots & \ldots & 0 \\
0 & \ldots & 0 & \widetilde{g} / C & -(g+2 \widetilde{g}) / C & \widetilde{g} / C \\
0 & 0 & \ldots & 0 & \widetilde{g} / C & -(g+2 \widetilde{g}) / C
\end{array}\right] \text {, }
$$

$H_{2}=-I_{N} / C, H_{3}=I_{N} / L$ and $H_{4}=-r I_{N} / L$, where $I_{N}$ is the $N \times N$ identity matrix. The $2 \times 2$ matrix $M$ takes the value $M_{1}$ when $v_{\text {th }}^{1}<v<v_{\text {th }}^{2}$ and $M_{2}$ otherwise, where

$$
M_{1}=\left[\begin{array}{cc}
(1-\widehat{g}) / C & -1 / C \\
1 & -\gamma
\end{array}\right], \quad M_{2}=\left[\begin{array}{cc}
-(1+\widehat{g}) / C & -1 / C \\
1 & -\gamma
\end{array}\right] .
$$

The $(2 N+2) \times 1$ vector $b(t)$ is given by $\alpha+\beta(t)$, where

$$
\alpha=\left[\begin{array}{lll}
0 & 0 & \ldots f
\end{array}\right]^{T}, \quad \beta(t)=\left[\begin{array}{lllllll}
I_{\mathrm{inj}, 1}(t) & \ldots & I_{\mathrm{inj}, N}(t) & 0 & \ldots 0 & I_{\mathrm{inj}, 2 N+1}(t) & 0
\end{array}\right]^{T},
$$

and $f$ is a $1 \times 2$ vector that takes the value $f_{1}$ for $v<v_{\text {th' }}^{1} f_{2}$ for $v_{\text {th }}^{1}<v<v_{\text {th }}^{2}$ and $f_{3}$ for $v>v_{\text {th }}^{2}$ :

$$
f_{1}=\left[\begin{array}{ll}
J / c & 0
\end{array}\right], \quad f_{2}=[(J-a) / c \quad 0], \quad f_{3}=[(J+1) / c \quad 0] .
$$


Note that the term $I_{\text {inj,2N+1 }}(t)$ in $\beta(t)$ is introduced to allow for the possibility of timedependent drive to the somatic compartment. With these definitions the general solution of (6) can be written by matching solutions of the form

$$
\begin{aligned}
z(t) & =G(t) z(0)+K(t) \alpha+\kappa(t), \\
G(t) & =\mathrm{e}^{A t}, \quad K(t)=\int_{0}^{t} G(s) \mathrm{d} s, \quad \kappa(t)=\int_{0}^{t} G(s) \beta(t-s) \mathrm{d} s,
\end{aligned}
$$

according to the rules for choosing $M$ and $f$, namely according to where the somatic voltage $v$ is in comparison to the two thresholds $v_{\text {th }}^{1,2}$.

\section{Mode-locked Solutions}

The phenomenon of mode-locking is well documented in the literature on the periodic forcing of nonlinear oscillators. It is most commonly studied in the context of the standard circle map (see for example [Boyland, 1986]). This map is known to support regions of parameter space where the rotation number (average rotation per map iterate) takes the value $p / q$, where $p, q \in \mathbb{Z}^{+}$. These regions are referred to as Arnol'd tongues. In a neural context mode-locked solutions are simply identically recurring firing patterns for which a neuron fires $p$ APs for every $q$ cycles of a periodic injected current. As discussed in Sec. 1, much (if not all) of the modeling work to date in this area has focused on point models, with most analytical progress being made for variants of the IF model (that typically do not have a realistic representation of the AP shape). However, the challenge of understanding mode-locking in spatially extended neuronal models that are also capable of generating realistic AP shapes is readily pursued for the model of Sec. 2. The key feature of this model that allows for explicit mathematical progress is its PWL nature. Consider again for the moment the periodic orbit of the original McKean model shown in Fig. 1, obtained for constant, rather than periodic, current injection. This periodic orbit is naturally divided into four parts, which we label by $\mu=1, \ldots, 4$. The orbit crosses each of the two thresholds $v_{\text {th }}^{1,2}$ only twice. For the periodic forcing of our soma-dendrite model it is also natural to expect solutions that can be described with this form of labeling. For clarity we shall focus only on the case that a periodic orbit with somatic voltage component like that of Fig. 1 is generated for $q$ cycles of periodic forcing. Thus we study a form of 1:q mode-locked state, though stress here that the ideas we present generalize to cover 
other more complicated $p: q$ solutions. On each part of the periodic solution we can construct the solution $z_{\mu}(t)=G_{\mu}(t) z_{\mu}(0)+K_{\mu}(t) \beta_{\mu}+\kappa_{\mu}(t)$, where $A_{\mu}$ and $b_{\mu}$ on each part of the phase space are determined according to the rules for choosing $M$ from $\left\{M_{1}, M_{2}\right\}$ and $f$ from $\left\{f_{1}, f_{2}, f_{3}\right\}$. For example, in region $\mu=4$ where $v<v_{\text {th }}^{1}$ we would choose $(M, f)=\left(M_{2}, f_{1}\right)$. The periodic orbit is naturally parametrised in terms of initial data $z_{1}(0)=z^{*}$, with all components of this vector as yet undetermined apart from the somatic voltage which is set to $v_{\mathrm{th}}^{1}$. In this case

$$
z_{\mu+1}(0)=G_{\mu}\left(T_{\mu}\right) z_{\mu}(0)+K_{\mu}\left(T_{\mu}\right) b_{\mu}+\kappa_{\mu}\left(T_{\mu}\right), \quad \mu=1,2,3 .
$$

The 'times-of-flight' $T_{\mu}$ are determined by solving the threshold crossing conditions at the soma: $v_{1}\left(T_{1}\right)=v_{\mathrm{th}}^{2}, v_{2}\left(T_{2}\right)=v_{\mathrm{th}}^{2}, v_{3}\left(T_{3}\right)=v_{\mathrm{th}}^{1}$, and $v_{4}\left(T_{4}\right)=v_{\mathrm{th}}^{1}$. A periodic solution can then be found by solving $z_{4}\left(T_{4}\right)=z_{1}(0)$, thus yielding $z^{*}$ and the period $T=\sum_{\mu=1}^{4} T_{\mu}$. The final condition that needs to be enforced to determine a 1:q modelocked solution is $T=2 \pi q / \omega$. Hence a 1:q mode-locked state is determined by the simultaneous solution of $2 N+6$ simultaneous nonlinear algebraic equations: $2 N+1$ periodicity constraints, 4 threshold crossing conditions, and one period constraint. The practical application of this procedure requires the calculation of $\kappa_{\mu}(t)$ for a given form of periodic current injection. For the remainder of this paper we shall work with the choice $I_{\mathrm{inj}, i}(t)=A_{i} \sin (\omega t+\phi)$. Here $\phi \in[0,2 \pi)$ is introduced as a convenience to define the phase of the mode-locked state with respect to the origin of time (which we fix with $\left.v_{1}(0)=v_{\text {th }}^{1}\right)$. The matrices $G_{\mu}(t), K_{\mu}(t)$ and $\kappa_{\mu}(t)$ are then easily constructed along the lines in [Coombes, 2008], though we do not bother with their presentation here. The resulting system of equations is then solved numerically in Matlab using fsolve (), for the elements of $z^{*}$ (excluding that of $v$ which is fixed at $v_{\text {th }}^{1}$ ), $T_{1}, \ldots, T_{4}$ and $\phi$. These $2 N+6$ parameters then completely determine the shape of the orbit according to $z_{\mu}(t)=G_{\mu}(t) z_{\mu}(0)+K_{\mu}(t) \beta_{\mu}+\kappa_{\mu}(t)$ and (13).

Since the soma-dendrite model is spatially extended it is natural to ask how the periodic behavior in each compartment varies across the chain. Indeed for global forcing (comprising an identical signal on each compartment) one might envisage a high degree of similarity between the dynamics of compartments (especially for compartments in the middle of a long chain where boundary effects are not strong). This similarity could be quantified by using an appropriate synchrony measure. However, of more interest is the spatial distribution of phases across the network. Indeed point forcing (of a single compartment) would lend itself more to the generation of a 
phase gradient. Thus a more natural measure to consider is one that distinguishes between standing and traveling waves. One such measure has already been introduced in [Marts et al., 2007] for the periodically forced planar Belousov-Zhabotinsky reaction, and we adopt this here. Defining the Fourier transform of the voltage in the dendritic compartments by $V_{j}(\Omega)(j=1, \ldots, N$, with spectral parameter $\Omega)$ then we sample this signal at the frequency of forcing to obtain the filtered signal $\Psi_{j} \mathrm{e}^{i \omega t}+$ cc with complex amplitude $\Psi_{j}=V_{j}(\omega)$. A plot of the pair $\left(\operatorname{Re} \Psi_{j}, \operatorname{Im} \Psi_{j}\right)$ defines a phase-plot that can be visually inspected to determine whether the pattern in the dendritic tree is more like a standing or traveling wave. To understand this last statement it is informative to consider a standing wave of the form $\cos (k j) \mathrm{e}^{i \omega t}$ for some real wave-number $k$ and $j \in \mathbb{Z}$. In this case the amplitude is wholly real and the phase-plot is simply a set of points on the real line covering the interval $[-1,1]$. For a traveling wave $\mathrm{e}^{i(\omega t-k j)}$ of constant amplitude the phase-plot is a set of points on the unit circle, and for one with a decaying amplitude (around some fixed value of $j$ ) points line up on a spiral. Thus if the phase plot is a set of points that lies near a line passing through the origin we shall call it a standing wave. If the points are closer to the unit circle we shall call it a traveling wave of uniform amplitude, whilst if they line up on a spiral we have a traveling wave with a decaying amplitude (which will be largest at the point of stimulation). A plot of a 1:3 mode-locked solution constructed according to the prescription above, for both global and point forcing is shown in Fig. 4. In the same plot we show the corresponding phase-plot, which nicely illustrates that global forcing favors standing waves and local forcing favors traveling waves.

\section{Arnol'd Tongues}

With an increase of the coupling amplitude from zero Arnol'd tongues in the standard circle map typically open as a wedge, centered at points in parameter space where the natural frequency of the oscillator is rational. In between tongues quasi-periodic behavior, emanating from irrational points on the amplitude/frequency axis, are observed. The tongue borders are defined in terms of instabilities of solutions with rational rotation number, and are thus defined in terms of either saddle-node or perioddoubling bifurcations. Since the map in this case is given it is straightforward to calculate the tongue structure in a two-dimensional parameter plane. In the more general 
setting of a periodically forced oscillator one is first required to integrate the differential equation model for the flow to find each iterate. The computational challenges associated with this task are exhaustively analyzed in the recent article by Schilder and Peckham [Schilder and Peckham, 2007]. For the case considered here we first consider a stroboscopic map which takes the form

$$
z(t) \mapsto z\left(t+\frac{2 \pi q}{\omega}\right) .
$$

The stability properties of a 1:q mode-locked solution then follow from an analysis of fixed points of this map. The linearisation of this map around a 1:q state yields the Jacobian $\Gamma$, defined by

$$
\Gamma=G_{4}\left(T_{4}\right) G_{3}\left(T_{3}\right) G_{2}\left(T_{2}\right) G_{1}\left(T_{1}\right) .
$$

If all the eigenvalues of $\Gamma$ have modulus less than unity, then the $1: q$ solution is asymptotically stable. We are thus led to the construction of the Arnol'd tongue structure in terms of i) a saddle-node bifurcation, where $\operatorname{det}\left(\Gamma-I_{2 N+2}\right)=0$, ii) a period doubling bifurcation where $\operatorname{det}\left(\Gamma+I_{2 N+2}\right)=0$, and iii) a Neimark-Sacker bifurcation where $\operatorname{det}(\Gamma)=1$ [Wiggins, 1990].

However this only accounts for instabilities of the stroboscopic map, and not those arising from the underlying flow. Since 1:q solutions have been defined in such a way that the somatic voltage crosses each of the two thresholds only twice then such solutions can be lost in grazing bifurcations, where a solution tangentially intersects with $v_{\mathrm{th}}^{1,2}$. This can happen in two different ways, and we are lead to two distinct types of grazing bifurcation, which we shall refer to as Type I and Type II. The condition for a Type I grazing bifurcation can be written $\mathrm{d} v /\left.\mathrm{d} t\right|_{t=T_{\mu}}=0$. The condition for a Type II graze takes the form $v\left(T^{*}\right)=v_{\text {th }}^{1,2}$ and $\mathrm{d} v /\left.\mathrm{d} t\right|_{t=T^{*}}=0$, for some time $0<T^{*}<T_{\mu}$. An example of an orbit at a Type II grazing bifurcation is shown in Fig. 5.

To define a tongue border in parameter space we must append the bifurcation conditions described above to the $2 N+6$ equations defining a 1:q state. For a border defined by an instability of the stroboscopic map or a Type I graze this means appending only one extra equation - thus if we leave one of the soma-dendrite parameters as free it can be used to solve this extra equation. For a Type II grazing bifurcation we must append two equations - one determining the grazing time $T^{*}$ and the other again fixing the position of the bifurcation in a parameter of the soma-dendrite model. In Fig. 6 we show a plot of the tongue structure obtained using the approach above for 
both a passive and quasi-active compartment model. In both examples it can be seen that the 1:q solutions occupy a significant fraction of the amplitude/frequency parameter space. For both global and point forcing (with the same amplitude and frequency) the tongue structures are broadly similar - though solutions within tongues do differ as described earlier, with standing waves preferred for global forcing and traveling waves for local forcing. Note that in the resonant case the tongues can appear to close over with increasing amplitude of forcing. However, this is due to a grazing bifurcation, which does not necessarily lead to a change in the number of APs generated in a cycle, and rather can just change the number of times that a threshold $v_{\text {th }}^{1,2}$ is crossed during an orbit.

As a confirmation of our tongue construction we performed brute force numerical simulations of the full soma-dendrite dynamical system in Matlab. As a measure of whether we are in a mode-locked state or not we look at the variability of the period of the system. This is done using a Poincare section at $v=v_{\mathrm{th}}^{1}$. When the orbit crosses the section in the direction from left to right in the phase-plane the $i$ th crossing time is recorded and stored as $T_{i}$. For a 1:q mode-locked state like that in Fig. 1 the instantaneous period $T_{i+1}-T_{i}$ is constant for all $i$. However for other solutions this is not the case. This suggests using the following measure to pick out the 1:q solutions we have studied analytically - namely we calculate the maximal variation of the instantaneous period, defined as $\max \left(T_{i+1}-T_{i}\right)-\min \left(T_{i+1}-T_{i}\right)$, for which a 1:q state would give zero. A plot of this measure is shown in the left part of Figs. 7 and 8. Here, a warm color does not necessarily indicate chaotic behavior, as it could equally well signify an aperiodic solution, a $p: q$ mode-locked solution or even a 1:q solution that does not cross each of the two thresholds exactly twice.

To probe further whether the dynamics between tongues is aperiodic or chaotic we also calculated the Lyapunov exponents of the system using MATDS (a MATLAB package for the study of dynamical systems) [Govorukhin, 2004], which implements the algorithm in [Wolf et al., 1985]. As can be seen from the overlay of our original 1:q tongue plot with direct simulations, as shown in Figs. 7 and 8, there is excellent agreement between theory and numerical experiments. As expected higher order $2: q+q^{\prime}$ orbits are found sandwiched between $1: q$ and $1: q^{\prime}$ orbits and indeed the usual ordering of $p: q$ tongues in circle maps seems to occur (organized according to a Farey sequence in $p / q$ ). We also find that the quasi-active dendrite generally has larger Lyapunov exponents than the passive dendrite, and more easily lends itself to parameter 
values that support chaotic motion.

\section{Discussion}

In this paper we have introduced an analytically tractable model of a spatially extended single neuron by coupling an active PWL soma model to a compartmental dendritic tree. Importantly this model inherits the ability of the McKean model to generate realistic AP shapes and further acknowledges the known role of dendrites in shaping neuronal output [Mainen and Sejnowski, 1996]. Only the soma is an intrinsic oscillator, the dendritic compartments being described by non-oscillatory dynamics that can be either passive or resonant. It is worth emphasising here that the quasi-active description of dendritic membrane that we have described is known to be important for fitting real neuronal data, such as that recorded from CA1 hippocampal cells [Coombes et al., 2007]. By focusing on the response to periodic forcing we have shown that this minimal model can be directly analyzed to understand emergent behavior and in particular the standing and traveling waves that correspond to modelocked states. The predicted Arnol'd tongue structure is in excellent agreement with direct numerical simulations. As the understanding of coupled neural networks has advanced with models of synaptically and gap-junction coupled point neuron models we now advocate an extension of this axo-somatic programme to networks with axo-dendritic interactions. The model we have introduced here is an obvious candidate for the basic building block of a theoretical programme. It can already be extended in two important biological ways whilst preserving its mathematically minimal character. Firstly, the McKean model can be replaced by any PWL caricature of an excitable membrane, of which there are several (see [Coombes, 2008] for a recent discussion). Secondly, arbitrary branched structures can be treated, according to the rules described in [Bressloff et al., 1996]. A programme along the above lines is currently being developed and will be reported upon elsewhere. Moreover the framework we have presented her is ideally suited to construct phase response curves [Ermentrout and Kopell, 1990] for spatially extended systems - a problem of particu-

lar interest to the experimental neuroscience community who routinely stimulate and record from different sites on the neuron. 


\section{List of Figures}

1 The phase plane for the McKean model has a nullcline with a piecewise linear cubic shape (dashed green line) corresponding to $\dot{v}=0$ and a linear one associated with $\dot{w}=0$ (dotted blue line). Parameters are $c=0.1, J=0.5, \gamma=0.5$ and $a=0.25$. The red line corresponds to a stable periodic orbit. . . . . . . . . . . . . . . . 20

2 An electrical diagram defining an 'LRC' circuit. Here $R$ represents the cell membrane resistance and $C$ its membrane capacitance. The electrical resistance $r$ and the inductance $L$ can be derived from a biophysical conductance based models with active currents, or fitted directly to subthreshold voltage data from experiments. . . . . . . . . . . .

3 A schematic of the soma-dendrite model. An active PWL McKean soma model with voltage $v$ is coupled to a chain of $N$ 'LRC' compartments, each with voltage $V_{i}$ and $i=1, \ldots, N$. The conductance that couples the soma to the first compartment is denoted by $\widehat{g}$, and the coupling between compartments is denoted by $\widetilde{g}$. The 'LRC' circuit is defined by the diagram in Fig. $2 . \quad \ldots \ldots \ldots \ldots \ldots \ldots$

4 Top left: A plot of $(v, w)$ for a 1:3 orbit in the soma-dendrite model with $N=100$ compartments and sinusoidal point forcing at $i=50$ with $A_{i}=$ 0.1 and $\omega=5.5$. The parameters of the McKean model are $c=0.1, J=$ $0.5, \gamma=0.5$ and $a=0.25$. The dendritic chain is passive, $L=0$ and $r \rightarrow \infty$, and the other parameters are $g=100, \widehat{g}=0.5, \widetilde{g}=5$ and $C=1$. Top right: the same with global forcing. Bottom left: the corresponding phase-plot showing a traveling wave with a decaying amplitude around the point of stimulation. Bottom right: the corresponding phase-plot showing a standing wave. . . . . . . . . . . . . .

$5 \quad$ Left: A 1:2 orbit with a Type II grazing point at $v=v_{\mathrm{th}}^{1}$. Here, $N=10$ and other parameters are as in Fig. 4. The sinusoidal drive is applied directly to the soma with amplitude 0.1 and frequency 3.55. Right: The solid line shows the trace of the somatic voltage plotted against time. The dashed line show $\sin (\omega t) \ldots \ldots \ldots \ldots \ldots \ldots \ldots$ 
6 Arnol'd tongues for 1:q mode-locked states in the soma-dendrite model of Fig. 4 with sinusoidal point forcing at $i=2$ for $N=10$. Left: A passive dendrite with $L=0$ and $r \rightarrow \infty$. Right: A quasi-active dendrite with $L=$ 100 and $r=1$. Solid (dotted) lines denote saddle-node (period-doubling) bifurcations of the stroboscopic map and dashed lines denote grazing bifurcations of the underlying flow. Note that in the resonant case (right) the 1:q tongues (which cross each of the two thresholds only twice) can close over with increasing amplitude of forcing. . . . . . . . . . .

7 Direct numerical simulations confirm the predicted Arnol'd tongue structure for the passive dendrite model of Fig. 6 left. Left: The maximal variation of the instantaneous period is color coded so that dark blue shows 1:q mode locking (that crosses each of the two thresholds exactly twice). Overlaid lines show the analytical tongue borders. Right: A color coded plot of the maximum Lyapunov exponent. . . . . . . . . . . . . 26

8 Left: Direct numerical simulations confirm the predicted Arnol'd tongue structure for the quasi-active dendrite model of Fig. 6 right. Right: In contrast to a passive dendrite model there are larger windows in parameter space capable of supporting chaotic behavior. . . . . . . . . 


\section{References}

[Aihara et al., 1984] Aihara, K., Matsumoto, G., and Ikegaya, Y. (1984). Periodic and non-periodic responses of a periodically forced Hodgkin-Huxley oscillator. Journal of Theoretical Biology, 109:249-269.

[Aihara et al., 1986] Aihara, K., Numajiri, T., Matsumoto, G., and Kotani, M. (1986). Structures of attractors in periodically forced neural oscillators. Physics Letters A, 116:313-317.

[Azouz and Gray, 2000] Azouz, R. and Gray, C. M. (2000). Dynamic spike threshold reveals a mechanism for synaptic coincidence detection in cortical neurons in vivo. Proceedings of the National Academy of Sciences USA, 97:8110-8115.

[Beierholm et al., 2001] Beierholm, U., Nielsen, C. D., Ryge, J., Alstrom, P., and Kiehn, O. (2001). Characterization of reliability of spike timing in spinal interneurons during oscillating inputs. Journal of Neurophysiology, 86:1858-1868.

[Boyland, 1986] Boyland, P. L. (1986). Bifurcations of circle maps: Arnol'd tongues, bistability and rotation intervals. Communications in Mathematical Physics, 106:353381.

[Bressloff, 1999] Bressloff, P. C. (1999). Resonantlike synchronization and bursting in a model of pulse-coupled neurons with active dendrites. Journal of Computational Neuroscience, 6:237-249.

[Bressloff and Coombes, 1997] Bressloff, P. C. and Coombes, S. (1997). Physics of the extended neuron. International Journal of Modern Physics B, 11:2343-2392.

[Bressloff et al., 1996] Bressloff, P. C., Dwyer, V. M., and Kearney, M. J. (1996). Sumover-paths approach to diffusion on trees. Journal of Physics A, 29:1881-1896.

[Brumberg and Gutkin, 2007] Brumberg, J. C. and Gutkin, B. (2007). Cortical pyramidal cells and non-linear oscillators: Experiment and spike-generation theory. Brain Research, 1171:122-137.

[Chik et al., 2004] Chik, D. T. W., Coombes, S., and Wang, Z. D. (2004). Clustering through post inhibitory rebound in synaptically coupled neurons. Physical Review E, 70 . 
[Coombes, 2008] Coombes, S. (2008). Neuronal networks with gap junctions: A study of piece-wise linear planar neuron models. SIAM Journal on Applied Dynamical Systems, to appear.

[Coombes and Bressloff, 1999] Coombes, S. and Bressloff, P. C. (1999). Mode-locking and Arnold tongues in integrate-and-fire neural oscillators. Physical Review E, 60:2086-2096.

[Coombes and Osbaldestin, 2000] Coombes, S. and Osbaldestin, A. H. (2000). Period adding bifurcations and chaos in a periodically stimulated excitable neural relaxation oscillator. Physical Review E, 62:4057-4066.

[Coombes et al., 2001] Coombes, S., Owen, M. R., and Smith, G. D. (2001). Modelocking in a periodically forced integrate-and-fire-or-burst neuron model. Physical Review E, 64:041914.

[Coombes et al., 2007] Coombes, S., Timofeeva, Y., Svensson, C.-M., Lord, G., Josić, K., Cox, S., and Colbert, C. (2007). Branching dendrites with resonant membrane: A "sum-over-trips" approach. Biological Cybernetics, 97:137-149.

[Dayan and Abbott, 2001] Dayan, P. and Abbott, L. F. (2001). Theoretical Neuroscience: Computational and Mathematical Modeling of Neural Systems. MIT Press.

[Doi and Sato, 1995] Doi, S. and Sato, S. (1995). The global bifurcation structure of the BVP neuronal model driven by periodic pulse trains. Mathematical Biosciences, 125:229-250.

[Ermentrout and Kopell, 1990] Ermentrout, G. B. and Kopell, N. (1990). Oscillator death in systems of coupled neural oscillators. SIAM Journal on Applied Mathematics, 50:125-146.

[Fellous et al., 2001] Fellous, J. M., Houweling, A. R., Modi, R. H., Rao, R. P. N., Tiesinga, P. H. E., and Sejnowski, T. J. (2001). Frequency dependence of spike timing reliability in cortical pyramidal cells and interneurons. Journal of Neurophysiology, 85:1782-1787.

[Fitzhugh, 1961] Fitzhugh, R. (1961). Impulses and physiological states in theoretical models of nerve membranes. Biophysical Journal, 1182:445-466. 
[Freeman and Johnson, 1982] Freeman, A. W. and Johnson, K. O. (1982). Cutaneous mechanoreceptors in macaque monkey: Temporal discharge patterns evoked by vibration, and a receptor model. Journal of Physiology, 323:21-41.

[Glass and Mackey, 1988] Glass, L. and Mackey, M. C. (1988). From Clocks to Chaos: The Rythms of Life. Princeton University Press.

[Govorukhin, 2004] Govorukhin, V. N. (2004). MATDS http://kvm.math.rsu.ru/matds/.

[Graham, 2004] Graham, B. (2004). Computational Neuroscience: Cortical Dynamics, chapter Dynamics of Storage and Recall in Hippocampal Associative Memory Networks. Springer.

[Huhn et al., 2005] Huhn, Z., Orbán, G., Erdi, P., and Lengyel, M. (2005). Theta oscillation-coupled dendritic spiking integrates inputs on a long time scale. Hippocampus, 15(7):950-62.

[Hunter et al., 1998] Hunter, J. D., Milton, J. G., Thomas, P. J., and Cowan, J. D. (1998). Resonance effect for neural spike time reliability. Journal of Neurophysiology, 80:14271438.

[Hutcheon et al., 1996] Hutcheon, B., Miura, R. M., and Puil, E. (1996). Models of subthreshold membrane resonance in neocortical neurons. Journal of Neurophysiology, 76:698-714.

[Hutcheon and Yarom, 2000] Hutcheon, B. and Yarom, Y. (2000). Resonance, oscillation and the intrinsic frequency preferences of neurons. Trends in Neurosciences, 23:216-222.

[Kaplan et al., 1996] Kaplan, D. T., Clay, J. R., Manning, T., Glass, L., Guevara, M. R., and Shrier, A. (1996). Subthreshold dynamics in periodically stimulated squid giant axons. Physical Review Letters, 76(21):4074-4077.

[Keener et al., 1981] Keener, J. P., Hoppensteadt, F. C., and Rinzel, J. (1981). Integrateand-fire models of nerve membrane response to oscillatory input. SIAM Journal on Applied Maths, 41(3):816-823. 
[Koch, 1984] Koch, C. (1984). Cable theory in neurons with active, linearized membranes. Biological Cybernetics, 50:15-33.

[Laing and Coombes, 2005] Laing, C. R. and Coombes, S. (2005). Mode locking in a periodically forced "Ghostbursting" neuron model. International Journal of Bifurcation and Chaos, 15:1433-1444.

[Lee and Kim, 2006] Lee, S.-G. and Kim, S. (2006). Bifurcation analysis of modelocking structure in a Hodgkin-Huxley neuron under sinusoidal current. Physical Review E, 73:041924.

[Lengyel et al., 2003] Lengyel, M., Szatmary, Z., and Erdi, P. (2003). Dynamically detuned oscillations account for the coupled rate and temporal code of place cell firing. Hippocampus, 13:700-714.

[Lin et al., 1999] Lin, A. L., Petrov, V., Swinney, H. L., Ardelea, A., and Carey, G. F. (1999). Pattern formation in continuous and coupled systems, chapter Resonant pattern formation in a spatially extended chemical system. Springer.

[Mainen and Sejnowski, 1995] Mainen, Z. F. and Sejnowski, T. J. (1995). Reliability of spike timing in neocortical neurons. Science, 268:1503-1506.

[Mainen and Sejnowski, 1996] Mainen, Z. F. and Sejnowski, T. J. (1996). Influence of dendritic structure on firing pattern in model neocortical neurons. Nature, 382:363366.

[Marts et al., 2007] Marts, B., Simpson, D. J. W., Hagberg, A., and Lin, A. L. (2007). Period doubling in a periodically forced Belousov-Zhabotinsky reaction. Physical Review E, 76:026213(1-6).

[McKean, 1970] McKean, H. P. (1970). Nagumo's equation. Advances in Mathematics, 4:209-223.

[Nagumo and Sato, 1972] Nagumo, J. and Sato, S. (1972). On a response characteristic of a mathematical neuron model. Kybernetik, 10:155-164.

[Pikovsky et al., 2001] Pikovsky, A., Rosenblum, M., and Kurths, J. (2001). Synchronization: A Universal Concept in Nonlinear Sciences. Cambridge University Press. 
[Rall, 1964] Rall, W. (1964). Neural Theory and Modeling, chapter Theoretical significance of dendritic trees for neuronal input-output relations. Stanford University Press.

[Rieke et al., 1997] Rieke, F., Warland, D., de Ruyter van Steveninck, R., and Bialek, W. (1997). Spikes: exploring the neural code. MIT Press.

[Sato and Doi, 1992] Sato, S. and Doi, S. (1992). Response characteristics of the BVP neuron model to periodic pulse inputs. Mathematical Biosciences, 112:243-259.

[Schilder and Peckham, 2007] Schilder, F. and Peckham, B. B. (2007). Computing Arnol'd tongue scenarios. Journal of Computational Physics, 220:932-951.

[Stuart et al., 2008] Stuart, G., Spruston, N., and Häusser, M., editors (2008). Dendrites. Oxford University Press, 2nd edition.

[Tonnelier, 2002] Tonnelier, A. (2002). The McKean's caricature of the FitzHughNagumo model I. The space-clamped system. SIAM Journal on Applied Mathematics, 63:459-484.

[Wiggins, 1990] Wiggins, S. (1990). Introduction to Applied Nonlinear Dynamical Systems and Chaos. Springer-Verlag, New York.

[Wolf et al., 1985] Wolf, A., Swift, J. B., Swinney, H. L., and Vastano, J. A. (1985). Determining Lyapunov exponents from a time series. Physica D, 16:285-317.

[Yoshino et al., 1999] Yoshino, K., Nomura, T., Pakdaman, K., and Sato, S. (1999). Synthetic analysis of periodically stimulated excitable and oscillatory membrane models. Physical Review E, 59(1):956-969. 


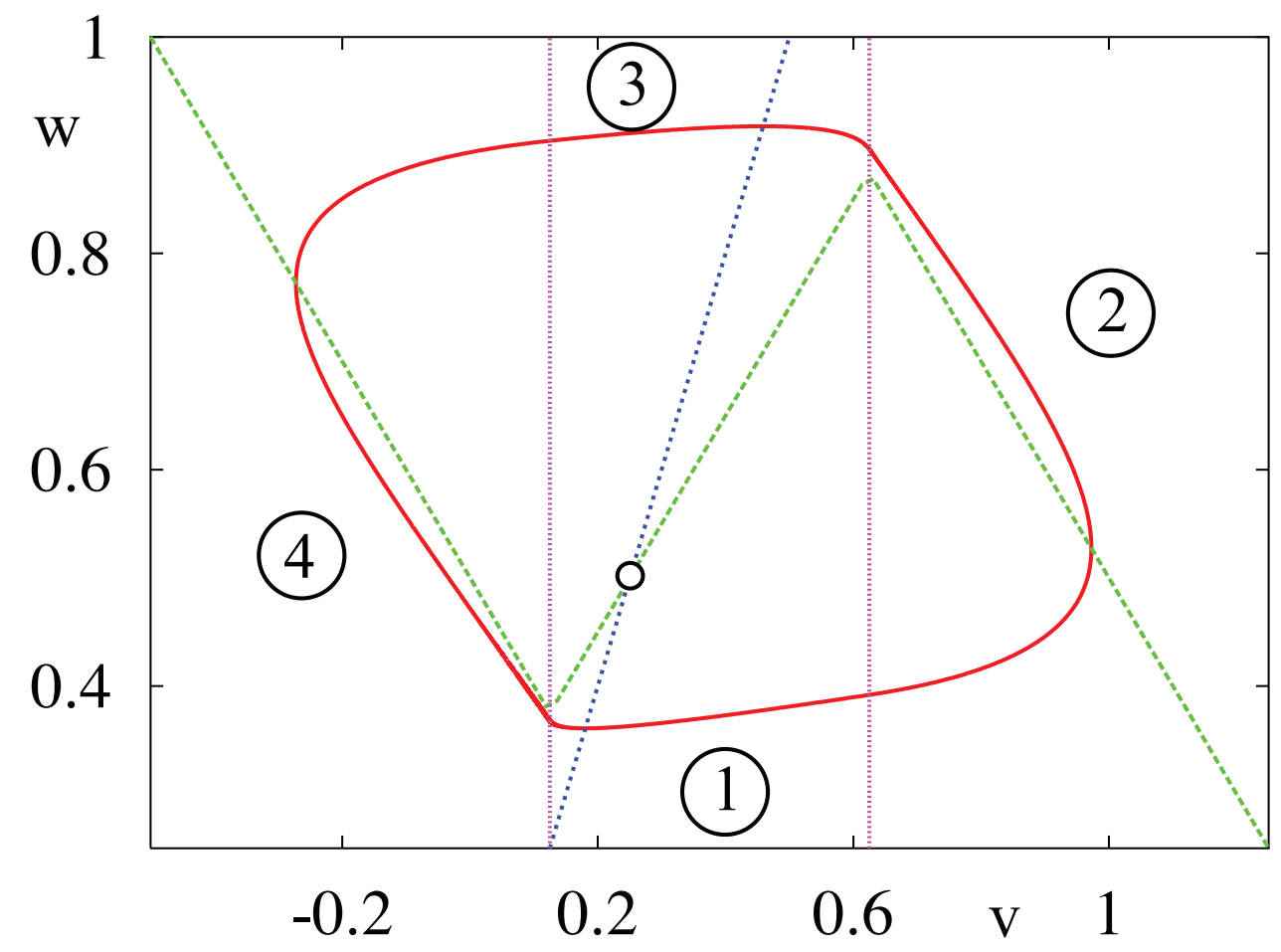

Figure 1: The phase plane for the McKean model has a nullcline with a piece-wise linear cubic shape (dashed green line) corresponding to $\dot{v}=0$ and a linear one associated with $\dot{w}=0$ (dotted blue line). Parameters are $c=0.1, J=0.5, \gamma=0.5$ and $a=0.25$. The red line corresponds to a stable periodic orbit. 


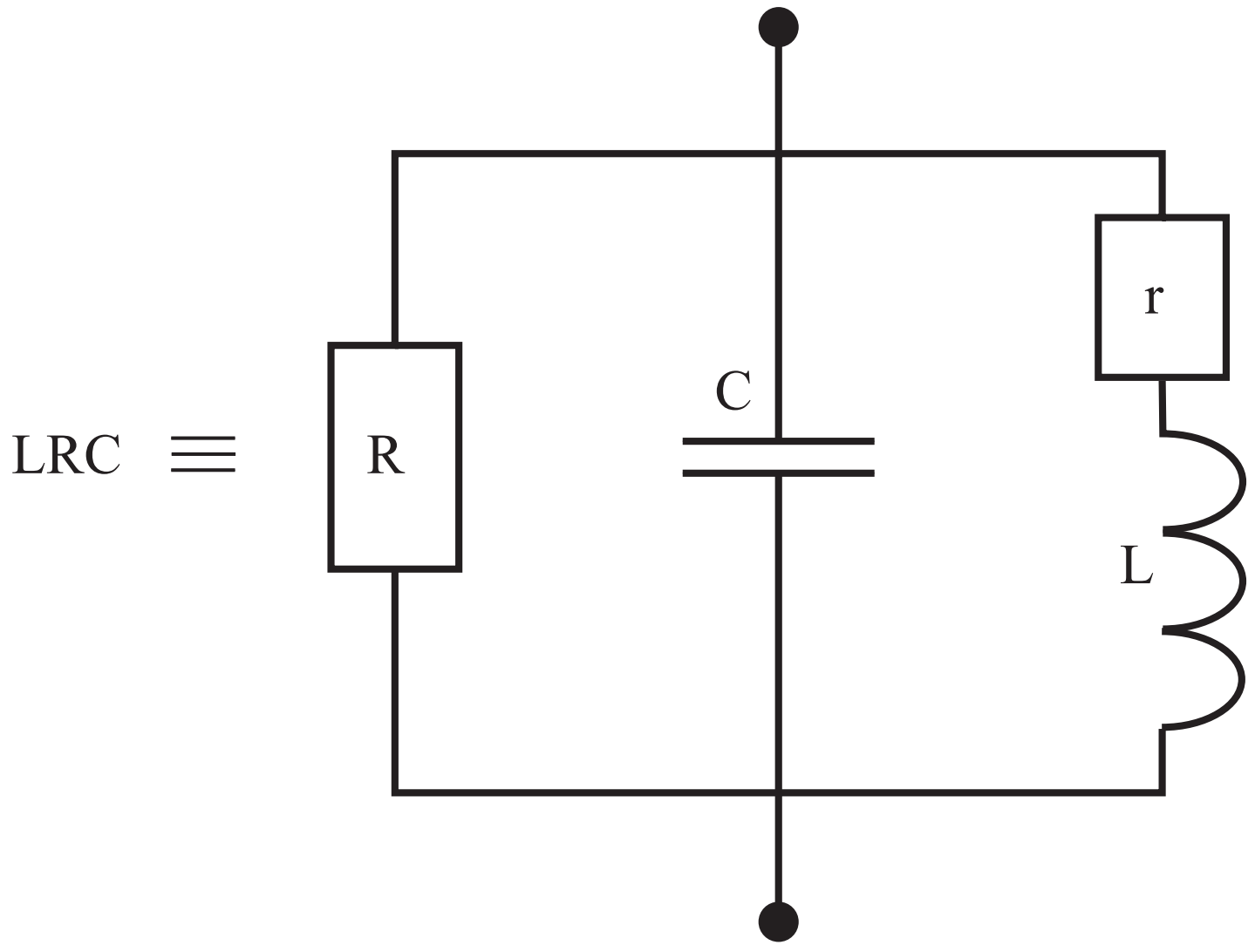

Figure 2: An electrical diagram defining an 'LRC' circuit. Here $R$ represents the cell membrane resistance and $C$ its membrane capacitance. The electrical resistance $r$ and the inductance $L$ can be derived from a biophysical conductance based models with active currents, or fitted directly to subthreshold voltage data from experiments. 


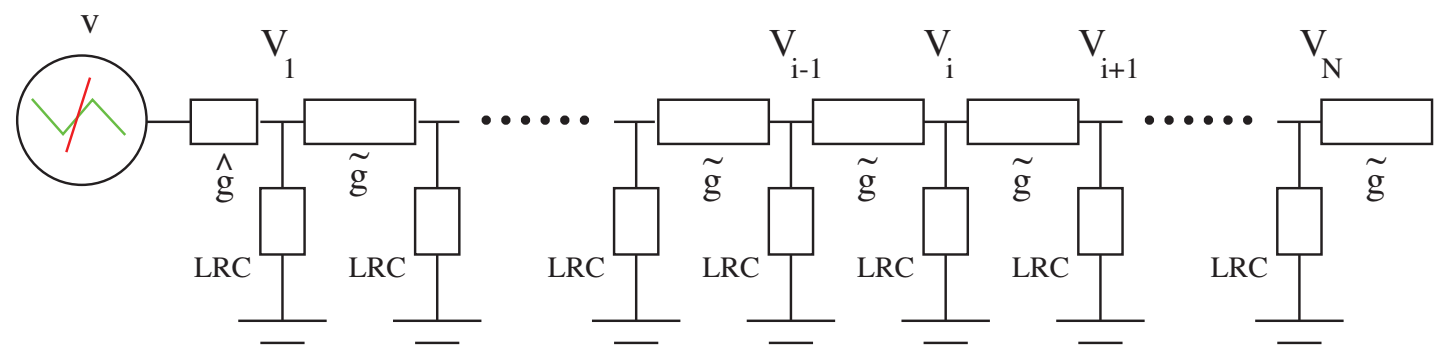

Figure 3: A schematic of the soma-dendrite model. An active PWL McKean soma model with voltage $v$ is coupled to a chain of $N$ 'LRC' compartments, each with voltage $V_{i}$ and $i=1, \ldots, N$. The conductance that couples the soma to the first compartment is denoted by $\widehat{g}$, and the coupling between compartments is denoted by $\widetilde{g}$. The 'LRC' circuit is defined by the diagram in Fig. 2 . 

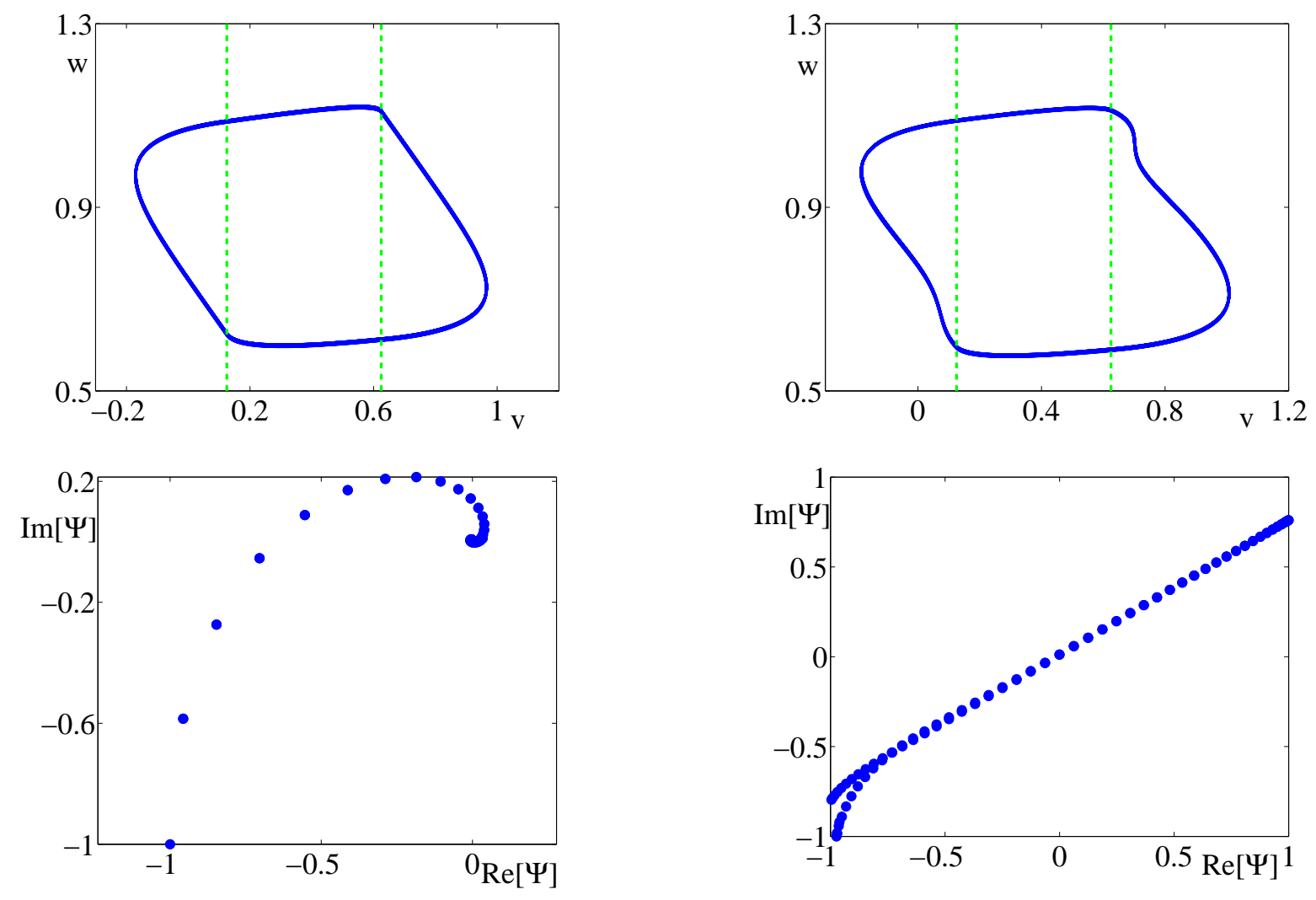

Figure 4: Top left: A plot of $(v, w)$ for a 1:3 orbit in the soma-dendrite model with $N=100$ compartments and sinusoidal point forcing at $i=50$ with $A_{i}=0.1$ and $\omega=$ 5.5. The parameters of the McKean model are $c=0.1, J=0.5, \gamma=0.5$ and $a=0.25$. The dendritic chain is passive, $L=0$ and $r \rightarrow \infty$, and the other parameters are $g=$ $100, \widehat{g}=0.5, \widetilde{g}=5$ and $C=1$. Top right: the same with global forcing. Bottom left: the corresponding phase-plot showing a traveling wave with a decaying amplitude around the point of stimulation. Bottom right: the corresponding phase-plot showing a standing wave. 

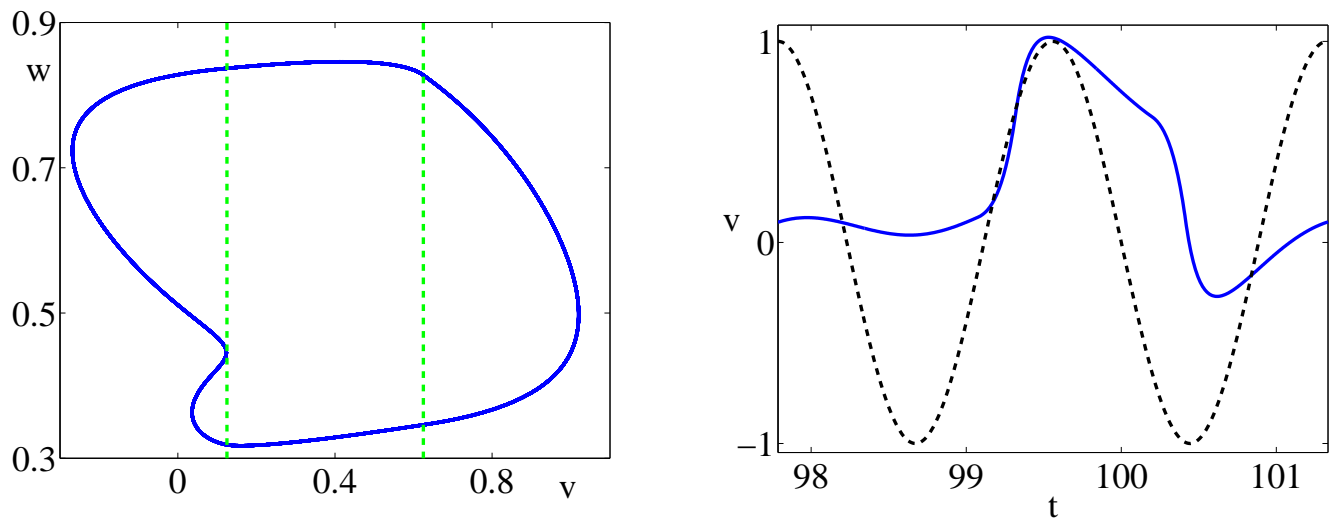

Figure 5: Left: A 1:2 orbit with a Type II grazing point at $v=v_{\text {th }}^{1}$. Here, $N=10$ and other parameters are as in Fig. 4. The sinusoidal drive is applied directly to the soma with amplitude 0.1 and frequency 3.55. Right: The solid line shows the trace of the somatic voltage plotted against time. The dashed line show $\sin (\omega t)$. 

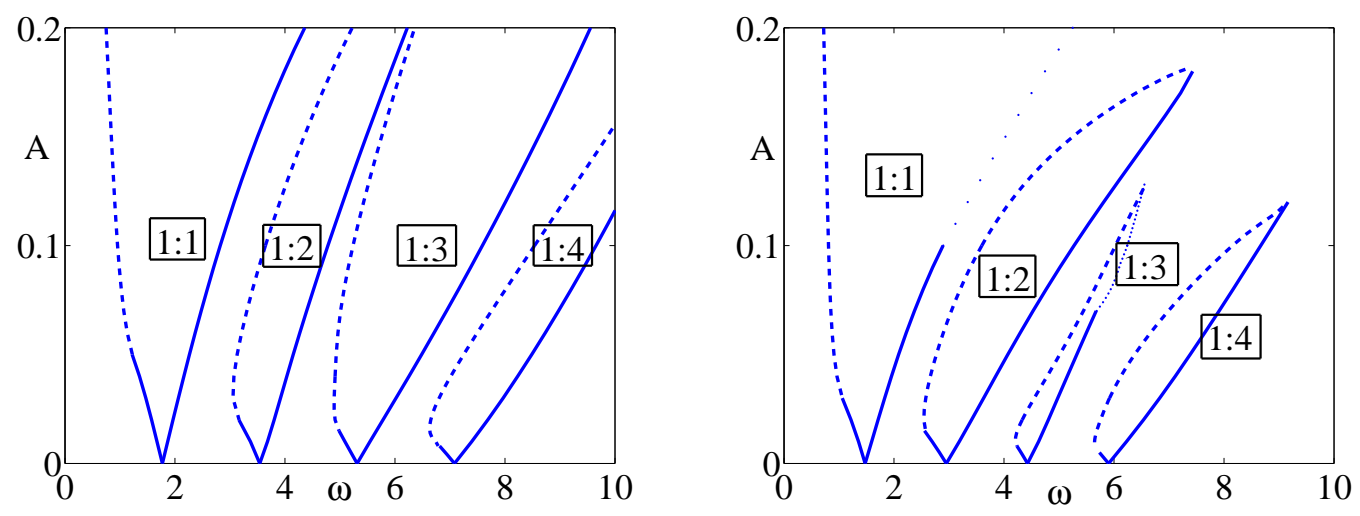

Figure 6: Arnol'd tongues for 1:q mode-locked states in the soma-dendrite model of Fig. 4 with sinusoidal point forcing at $i=2$ for $N=10$. Left: A passive dendrite with $L=0$ and $r \rightarrow \infty$. Right: A quasi-active dendrite with $L=100$ and $r=1$. Solid (dotted) lines denote saddle-node (period-doubling) bifurcations of the stroboscopic map and dashed lines denote grazing bifurcations of the underlying flow. Note that in the resonant case (right) the 1:q tongues (which cross each of the two thresholds only twice) can close over with increasing amplitude of forcing. 

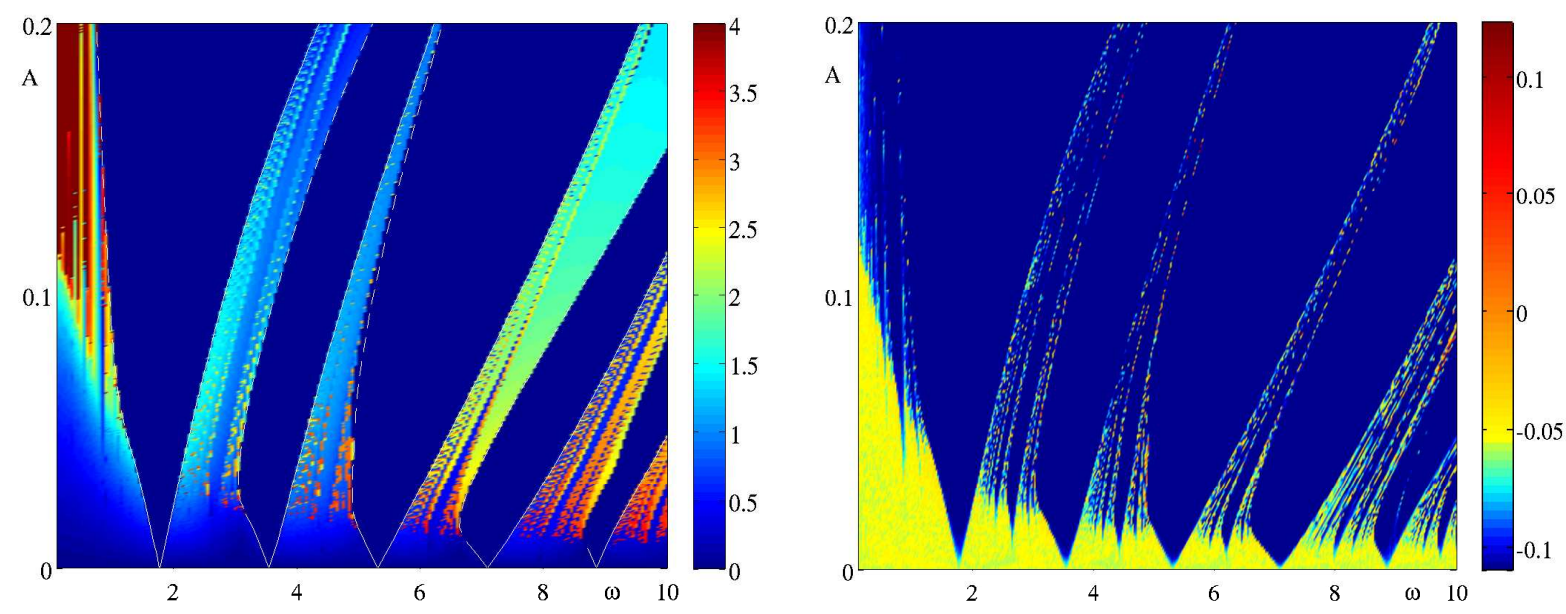

Figure 7: Direct numerical simulations confirm the predicted Arnol'd tongue structure for the passive dendrite model of Fig. 6 left. Left: The maximal variation of the instantaneous period is color coded so that dark blue shows 1:q mode locking (that crosses each of the two thresholds exactly twice). Overlaid lines show the analytical tongue borders. Right: A color coded plot of the maximum Lyapunov exponent. 

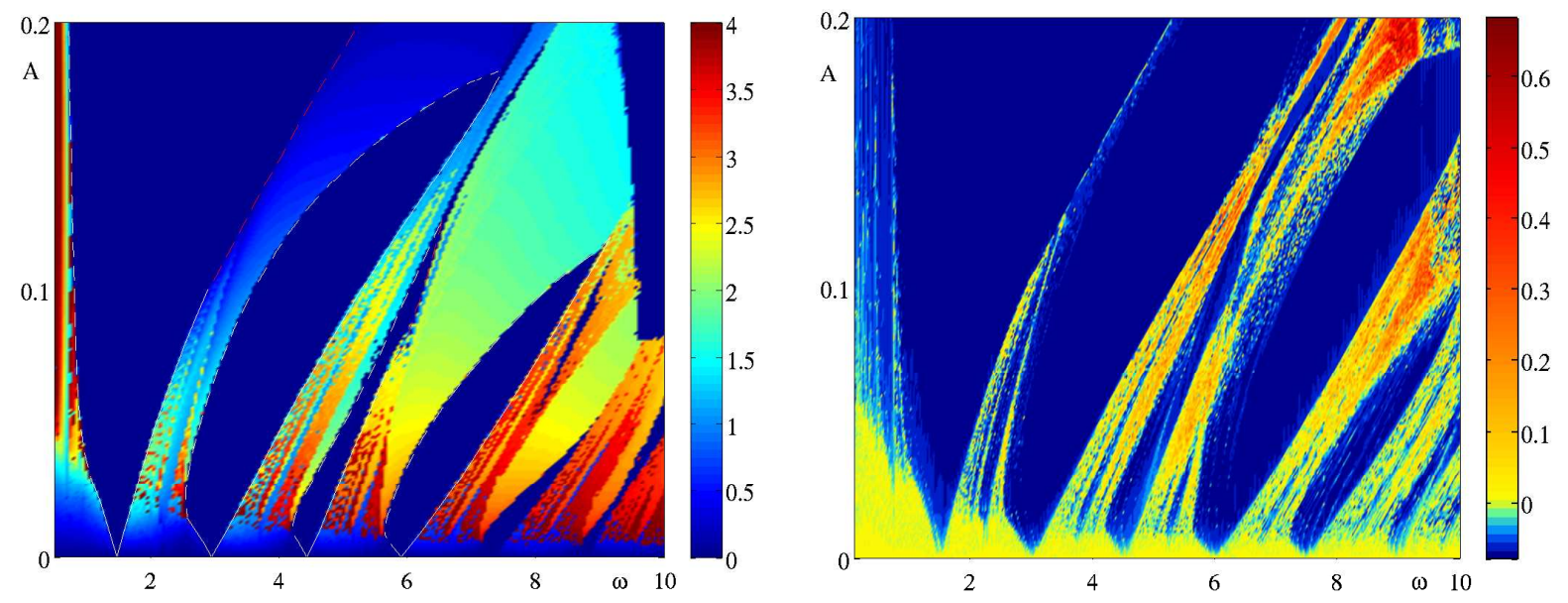

Figure 8: Left: Direct numerical simulations confirm the predicted Arnol'd tongue structure for the quasi-active dendrite model of Fig. 6 right. Right: In contrast to a passive dendrite model there are larger windows in parameter space capable of supporting chaotic behavior. 